\title{
The crystal structures of semi-synthetic aequorins
}

\author{
SACHIKO TOMA, ${ }^{1,5}$ KHOON TEE CHONG,${ }^{1}$ ATSUSHI NAKAGAWA, ${ }^{1}$ \\ KATSUNORI TERANISHI, ${ }^{2}$ SATOSHI INOUYE, ${ }^{3}$ AND OSAMU SHIMOMURA ${ }^{4}$ \\ ${ }^{1}$ Institute for Protein Research, Osaka University, Suita, Osaka 565-0871, Japan \\ ${ }^{2}$ Faculty of Bioscience, Mie University, Tsu-shi, Mie 514-8507, Japan \\ ${ }^{3}$ Yokohama Research Centre, Chisso Corporation, Kanazawa-ku, Yokohama 236-8605, Japan \\ ${ }^{4}$ The Photoprotein Laboratory, Falmouth, Massachusetts 02540, USA
}

(Received August 26, 2004; Final Revision November 3, 2004; AccePted November 3, 2004)

\begin{abstract}
The photoprotein aequorin emits light by an intramolecular reaction in the presence of a trace amount of $\mathrm{Ca}^{2+}$. Semi-synthetic aequorins, produced by replacing the coelenterazine moiety in aequorin with the analogues of coelenterazine, show widely different sensitivities to $\mathrm{Ca}^{2+}$. To understand the structural basis of the $\mathrm{Ca}^{2+}$-sensitivity, we determined the crystal structures of four semi-synthetic aequorins $(c p-, i-, b r$ - and $n$-aequorins) at resolutions of 1.6-1.8 $\AA$. In general, the protein structures of these semi-synthetic aequorins are almost identical to native aequorin. Of the four EF-hand domains in the molecule, EF-hand II does not bind $\mathrm{Ca}^{2+}$, and the loop of EF-hand IV is clearly deformed. It is most likely that the binding of $\mathrm{Ca}^{2+}$ with EF-hands I and III triggers luminescence. Although little difference was found in the overall structures of aequorins investigated, some significant differences were found in the interactions between the substituents of coelenterazine moiety and the amino acid residues in the binding pocket. The coelenterazine moieties in $i-, b r$-, and $n$-aequorins have bulky 2-substitutions, which can interfere with the conformational changes of protein structure that follow the binding of $\mathrm{Ca}^{2+}$ to aequorin. In $c p$-aequorin, the cyclopentylmethyl group that substitutes for the original 8-benzyl group does not interact hydrophobically with the protein part, giving the coelenterazine moiety more conformational freedom to promote the light-emitting reaction. The differences of various semi-synthetic aequorins in $\mathrm{Ca}^{2+}$-sensitivity and reaction rate are explained by the capability of the involved groups and structures to undergo conformational changes in response to the $\mathrm{Ca}^{2+}$ binding.
\end{abstract}

Keywords: active sites; conformational changes; radiation damage; crystallography; photoprotein; EFhand; $\mathrm{Ca}^{2+}$-binding protein; bioluminescence

The photoprotein aequorin obtained from the jellyfish Aequorea aequorea emits light in the presence of $\mathrm{Ca}^{2+}$ (Shimomura et al. 1962). Because of its high sensitivity to $\mathrm{Ca}^{2+}$ and biological harmlessness, aequorin is widely used as a $\mathrm{Ca}^{2+}$ indicator in various biological systems. Aequorin is a complex of apoaequorin (apoprotein), coelenterazine, and molecular oxygen. When a trace amount of $\mathrm{Ca}^{2+}$ is

Reprint requests to: Atsushi Nakagawa, Institute for Protein Research, Osaka University, 3-2 Yamadaoka, Suita, Osaka 565-0871, Japan; e-mail: atsushi@protein.osaka-u.ac.jp; fax: +81-6-6879-4313.

${ }^{5}$ Present address: Faculty of Pharmaceutical Sciences, Kumamoto University, 5-1 Oe-honmachi, Kumamoto 862-0973, Japan.

Article published online ahead of print. Article and publication date are at http://www.proteinscience.org/cgi/doi/10.1110/ps.041067805. added, aequorin emits blue light $\left(\lambda_{\max } 465 \mathrm{~nm}\right)$ by an intramolecular reaction, decomposing into apoaequorin, coelenteramide, and $\mathrm{CO}_{2}$ (Shimomura and Johnson 1973, 1975). Apoaequorin can be regenerated into aequorin by treatment with coelenterazine and oxygen in the absence of $\mathrm{Ca}^{2+}$; in the presence of $\mathrm{Ca}^{2+}$, apoaequorin catalyzes the luminescent oxidation of coelenterazine resulting in a dim, continuous glow (Shimomura and Johnson 1975).

Recently, the crystal structure of native aequorin (PDB ID: 1EJ3) was determined at $2.4 \AA$ resolution (Head et al. 2000). According to the report, the crystallographic asymmetric unit of the crystals consists of two aequorin molecules, and each molecule of aequorin contains four helixloop-helix "EF-hand" motifs, of which three of them (EF- 
hands I, III, and IV) can bind $\mathrm{Ca}^{2+}$ (Head et al. 2000). Luminometric titration of aequorins with $\mathrm{Ca}^{2+}$ showed that two $\mathrm{Ca}^{2+}$ ions are needed to trigger the luminescence reaction, and the affinity of aequorin for these first two $\mathrm{Ca}^{2+}$ ions is about 20 times higher than that for the third $\mathrm{Ca}^{2+}$ ion (Shimomura 1995; Shimomura and Inouye 1996). The coelenterazine and molecular oxygen in the binding pocket of aequorin exist in their bound form, a hydroperoxide, and the terminal oxygen atom of the hydroperoxide group is stabilized by a hydrogen bonding network involving the phenolic $\mathrm{OH}$ of Tyr184 (Head et al. 2000). It has been suggested that the light-emitting reaction starts when the binding of $\mathrm{Ca}^{2+}$ disrupts those interactions (Head et al. 2000).

Coelenterazine, an imidazopyrazinone ( $7 H$-imidazo[1,2- $\alpha]$ pyrazin-3-one), is widely distributed in bioluminescent marine organisms as a luciferin (substrate for luciferase) or as a component of various $\mathrm{Ca}^{2+}$-sensitive photoproteins, such as aequorin and obelin (Shimomura et al. 1980; Thomson et al. 1997). In an effort to improve the light-emitting properties of aequorin, semi-synthetic aequorins were produced by replacing the coelenterazine moiety in aequorin with various analogues of coelenterazine (Shimomura et al. 1988, 1989, 1990, 1993a,b). The semi-synthetic aequorins obtained with the analogues containing a 2- or 8-substituent showed widely different $\mathrm{Ca}^{2+}$-sensitivities and light-emitting characteristics. On the other hand, the $p$-hydroxyphenyl group at the 6-position of coelenterazine appears to be required in its intact form for the formation of aequorins, and the analogues modified at this position did not form semisynthetic aequorins with appreciable luminescence activity. To elucidate the mechanisms underlying the luminescence characteristics of semi-synthetic aequorins, we determined the atomic structures of four semi-synthetic aequorins, $c p-$, $b r-, i$, and $n$-aequorins, that contain a 2 - or 8 -substituent in their coelenterazine moiety.

In this paper, we used the previous sequence-numbering system (Head et al. 2000) to avoid confusion, and ordinary recombinant aequorin is called "native aequorin" to differentiate it from recombinant semi-synthetic aequorin.

\section{Results and Discussion}

\section{Overall structures of semi-synthetic aequorins}

In this study, the structures of four semi-synthetic aequorins, $c p-, i-, b r-$, and $n$-aequorins, were determined. These semi-synthetic aequorins contain the coelenterazine analogues that have substitutions at the $\mathrm{C} 2$ or $\mathrm{C} 8$ position (Fig. 1). $c p$-Aequorin, which exhibits a higher $\mathrm{Ca}^{2+}$ sensitivity compared to native aequorin, contains a cyclopentylmethyl group at the $\mathrm{C} 8$ position of its coelenterazine moiety

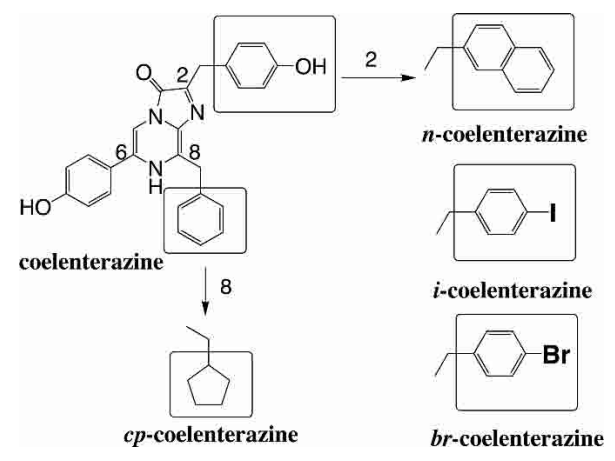

Figure 1. Chemical structures of coelenterazine and coelenterazine analogs.

(Shimomura et al. 1993b). On the other hand, $i-, b r-$, and $n$-aequorins, which all have lower $\mathrm{Ca}^{2+}$ sensitivities, contain a $p$-iodobenzyl group, a $p$-bromobenzyl group, and a $\beta$-naphthylmethyl group, respectively, substituted at the $\mathrm{C}-2$ position of the coelenterazine (Shimomura et al. 1993b).

Like the crystals of native aequorin, the crystals of these four semi-synthetic aequorins are composed of crystallographic asymmetric units, each containing two molecules of semi-synthetic aequorins, molecule $\mathrm{A}$ and molecule $\mathrm{B}$. In native aequorin, the loop region of EF-hand IV in molecule A appears to have two conformations. However, in semisynthetic aequorins, the molecules $\mathrm{A}$ and $\mathrm{B}$ have almost identical structures, and EF-hand IV has only one conformation corresponding to the molecule $\mathrm{B}$ of native aequorin (Fig. 2A).

The apoprotein used in this study is a recombinant protein with a modified $\mathrm{N}$-terminal sequence, ANSKL, in contrast to the wild-type apoprotein, which has VKL as the N-terminal sequence. The $\mathrm{N}$-terminal region of native aequorin is disordered, whereas the $\mathrm{N}$-terminal regions of the molecules $\mathrm{A}$ of $c p-, i-$, and $b r$-aequorins and of both the molecules A and $\mathrm{B}$ of $n$-aequorin, are clearly shown in the electron density maps.

Among all the structures examined, no significant difference was observed in the conformations of the amino acid residues in the binding pocket, or in the position of the coelenterazine moiety (Fig. 2A). The superposition of the molecules A of native, $c p-, i-$, and $b r$-aequorins onto the molecule A of $n$-aequorin (data1) gave root mean square (r.m.s.) deviations of only $0.7 \AA, 0.3 \AA, 0.4 \AA$, and $0.2 \AA$, respectively, considering all atoms. This result is unexpected considering the remarkable differences in the $\mathrm{Ca}^{2+}$ sensitivities of these aequorins, as well as the distinct differences in the chemical structures of the coelenterazine analogues involved. However, some significant differences were found in the interactions between the coelenterazine analogues and the amino acid residues in the binding pockets, as described below. 

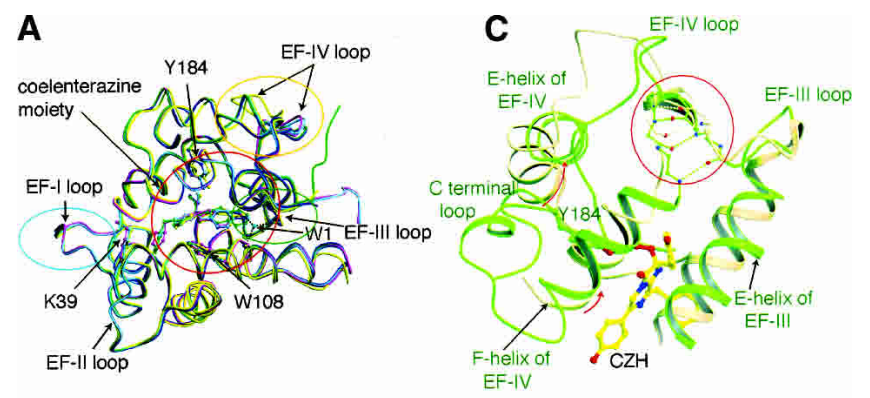

B

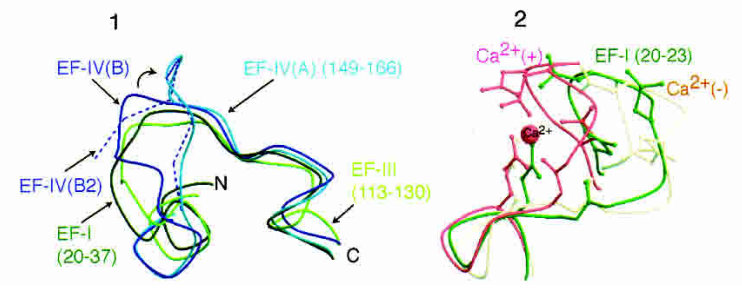

Figure 2. Structural comparison of native aequorin, semi-synthetic aequorins, and calmodulin. (A) Superimposed structures of molecule A of native aequorin and semi-synthetic aequorins. Native, $c p-, b r-, i-$, and $n$-aequorin are shown in yellow, pink, blue, sky blue, and green, respectively. Apoaequorins are shown as $\mathrm{C} \alpha$ trace models. Coelenterazine moieties are drawn as ball-and-stick models with same colors as above. The coelenterazine moiety and the loop region of EF-hands I, III, and IV are surrounded by red, light blue, green, and orange circles, respectively. W1 in native aequorin is drawn as a sphere model in cyan. The $\mathrm{OH}$ group of the 2-substituent of native coelenterazine is stabilized by a hydrogen-bonding network mediated by W1. W1 is absent in $b r-, i-$, and $n$-aequorin. The cyclopentyl group at the $\mathrm{C} 8$ position of $c p$-coelenterazine can make neither a stacking interaction with Lys39 nor a $\pi-\pi$ interaction with Trp108. This figure was prepared with MolScript (Kraulis 1991) and Raster3D (Merrit and Bacon 1997). (B) Superimposed structures of EF-hands I, III, and IV of aequorin. (1) The superimposed structures of EF-hands I (shown in sky blue circle in Fig. 2A: 20-37), III (shown in green circle in Fig. 2A: 113-130), and IV (shown in orange circle in Fig. 2A: 149-166). EF-hand IV in molecule A is colored cyan and EF-hand IV in molecule B is colored blue. EF-hands I and III are colored green and yellow-green, respectively. The blue dashed line, EF-IV(B2), is the loop structure of EF-hand-IV(B) superimposed onto the same region of EF-IV(A). (2) The superimposed structures of the EF-hand I loop (residues 20-37) of aequorin (green) and $\mathrm{Ca}^{2+}$-bound (deep pink) and $\mathrm{Ca}^{2+}$-unbound (cream) calmodulin. The $\mathrm{Ca}^{2+}$ is drawn as a sphere. All figures were prepared with MolScript (Kraulis 1991). (C) Structure comparison between aequorin and calmodulin. The superimposed structures of aequorin (yellow-green) and $\mathrm{Ca}^{2+}$-unbound calmodulin (cream). The traced region of aequorin is from Thr103 to Pro189. The traced region of calmodulin is from Ser81 to Lys148. The coelenterazine moiety $(\mathrm{CZH})$ is drawn as a ball-and-stick model. Dashed lines appeared in the inside of the red circle are main-chain interactions, green dotted lines are interactions in aequorin, and cream ones are interactions in calmodulin.

The structure of peroxide group bound to coelenterazine moiety

Recently, the structures of aequorin (Head et al. 2000), obelin (PDB ID: 1EL4; Liu et al. 2000), obelin bound to $\mathrm{Ca}^{2+}$ (PDB ID: 1QV1; Liu et al. 2003), and the W92F obelin mutant (PDB ID: 1JF2; Deng et al. 2001) were solved at resolutions of $2.3 \AA, 1.1 \AA, 1.7 \AA$, and $1.72 \AA$, respectively. In the structure of native aequorin, it was shown that a peroxide group $(\mathrm{O}-\mathrm{O})$ was attached to the $\mathrm{C} 2$ atom of the coelenterazine moiety, although the electron density of the terminal oxygen of the peroxide was weak (Head et al. 2000). Similar investigations on the obelin structure have provided conflicting results; the $\mathrm{C} 2$ atom was found to be in a mono-oxide form in one study (Liu et al. 2000), whereas the same $\mathrm{C} 2$ atom was reported to be peroxidized when the W92F obelin structure was solved (Deng et al. 2001). According to another report (Liu et al. 2003), obelin partially bound to $\mathrm{Ca}^{2+}$ exhibited a clear electron density for the second oxygen atom of the peroxide group. The report claimed that the binding of $\mathrm{Ca}^{2+}$ increased the structural rigidity of obelin, consequently decreasing the mobility of the $\mathrm{O}$ atom (Liu et al. 2003). In the present study, the electron density corresponding to the $\mathrm{C} 2$ peroxide group widely varied in each semi-synthetic aequorin. With $c p$ aequorin, the terminal oxygen of the peroxide was completely missing in the electron density map. With $i$ - and $b r$-aequorins, the terminal oxygen was shown only as weak density. Only $n$-aequorin gave an electron density map that clearly shows the terminal oxygen.

In the previous paper on the structure of native aequorin (Head et al. 2000), a crystal that had been irradiated with X rays retained about half of the peroxide in the intact form, as judged from the electron density. The irradiated crystal emitted $<50 \%$ of the light emitted by the crystal before irradiation, thus implying a correlation between the damage of peroxide and the loss of luminescence activity, both caused by X-ray irradiation. In the present study, we confirmed that X-ray irradiation causes the decrease in electron density of the terminal oxygen atom using $n$-aequorin. $n$ Aequorin was more favorable for crystallography than other semi-synthetic aequorins; its crystals grew to larger sizes and gave higher resolutions with good reproducibility. We collected the reflection data sets with different exposure times. The structures were refined using the $\mathrm{C} 2$ mono-oxide form of $n$-coelenterazine as a model. In the 1 -sec structure, the peak of $\sigma_{\mathrm{A}}$-weighted $m \mathrm{Fo}-D \mathrm{Fc}$ map clearly showed the electron density that corresponds to the terminal oxygen at the $2.0 \sigma$ level. Furthermore, the height of this peak varied with the exposure time (Fig. 3). The same phenomenon was observed for $i$ - and $b r$-aequorins (data not shown). The radiation damage depended on the volume of the crystal, the intensity of $\mathrm{X}$ ray, and exposure time. The height of the electron density of the terminal oxygen varied each time depending on the crystal used, even with the same semisynthetic aequorin and with the same exposure time. These results indicate the partial elimination of the terminal oxygen of the peroxide by X-ray irradiation. The discrepancy concerning the peroxide group in the obelin structure (Liu et al. 2000; Deng et al. 2001) must be caused by the ability 
A

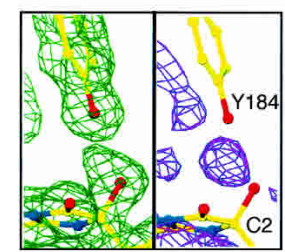

2Fo-Fc map Fo-Fc map

$n$-aequorin (data1)

$1 \mathrm{sec} /$ frame
B

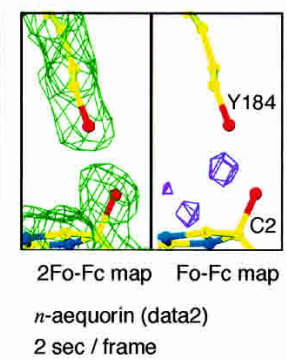

C

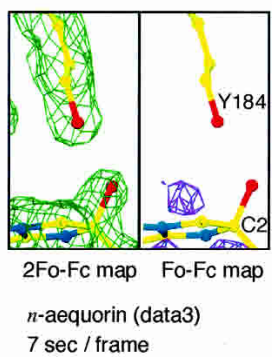

Figure 3. The electron-density map around the coelenterazine moiety in $n$-aequorin (data1, 2, and 3). (A) $n$-aequorin (data1) ( $1 \mathrm{sec}$ per frame), (B) $n$-aequorin (data2) (2 sec per frame), $(C) n$-aequorin (data3) $(7 \mathrm{sec}$ per frame). The label "Y184" corresponds to the Tyr184 residue and the label "C2" corresponds to $\mathrm{C} 2$ atom of the coelenterazine moiety. The green chicken wire map represents the $\sigma_{\mathrm{A}}$-weighted $2 m \mathrm{Fo}-D \mathrm{Fc}$ maps countered at 1.0 times the r.m.s. deviation of the map. These maps were calculated with the mono-oxide model. The violet chicken wire map represents the $\sigma_{\mathrm{A}^{-}}$ weighted $m$ Fo- $D$ Fc maps countered at 2.0 times the r.m.s. deviation, calculated with the mono-oxide model. Figures were prepared with program O (Jones et al. 1991) and Molray (Harris and Jones 2001).

of X-ray irradiation to break off the terminal oxygen atom of the peroxide group.

\section{Calcium-binding ability}

The superposition of the loop structures of EF-hands I, III, and IV in the four semi-synthetic aequorins and native aequorin revealed that the loop of EF-hand IV is significantly deformed (Fig. 2B1). In native aequorin, the loop region of EF-hand IV has two structurally similar but slightly different conformations (Fig. 2B1). A comparison of the sequence alignments of the EF-hands of native aequorin with calmodulin suggests that the coordination manner of the residues involved in the binding of $\mathrm{Ca}^{2+}$ might be identical in these two proteins. The loop structures of EF-hands I and III are similar (Fig. 2B1), and the conformation of the loop region involved is an intermediate state between the $\mathrm{Ca}^{2+}$ bound form and $\mathrm{Ca}^{2+}$-free form of calmodulin (PDB ID: 1CLL, Chattopadhyaya et al. 1992;PDB ID: 1CFC, Kuboniwa et al. 1995'; Fig. 2B2). The relative positions of the loops of EF-hands III and IV in aequorin are the same as those in calmodulin, except for some differences in the inter-helical angles. In fact, the relative positions of these loops are fixed by the main-chain hydrogen bonds, there being one additional main-chain interaction in aequorin compared to the calmodulin structure (Fig. 2C).

Relative to the structure of calmodulin, the F-helix of EF-hand IV is pushed aside by the 2-substituent of coelenterazine, and the E-helix of EF-hand IV is also detruded by the C-terminal loop that contains Tyr184 (Fig. 2C). For these reasons, the interaction between the EF-hand loops of III and IV in aequorins would be stronger than in calmod-

ulin, and the loop of EF-hand IV is consequently deformed. Based on these observations, it is suggested that EF-hands I and III bind $\mathrm{Ca}^{2+}$ more efficiently and strongly than EFhand IV in the triggering step of the luminescence reaction.

\section{C2 substitution}

In the structures of $i-, b r-$, and $n$-aequorins, which have low $\mathrm{Ca}^{2+}$ sensitivities, the water molecule (W1) that exists in the binding pocket of native aequorin is missing (Fig. 2A). Apparently, the space of $\mathrm{W} 1$ is replaced by the bulky 2-substituent of the coelenterazine analogues. In the structures of native aequorin and $c p$-aequorin, W1 makes hydrogen bonds with the phenolic $\mathrm{OH}$ of the 2-substituent, the $\mathrm{O} \gamma$ atom of Thr166, and the carbonyl oxygen of Ile105 (Fig. $2 \mathrm{~A}$ ). The volumes occupied by the bulky 2 -substituents are larger than that of the $p$-hydroxybenzyl group. Moreover, these bulky groups were inserted between the F-helix of EF-hand III and the E-helix of EF-hand IV. Thus, these bulky groups may hinder the conformational changes of EF-hand III that result upon the binding of $\mathrm{Ca}^{2+}$.

The main role of $\mathrm{W} 1$ in the molecules of native aequorin is considered to be the stabilization of coelenterazine moiety (Head et al. 2000). The hydrogen-bond network mediated by $\mathrm{W} 1$ would be easily broken during the rearrangement initiated by the binding of $\mathrm{Ca}^{2+}$. In the cases of $c p$-aequorin and native aequorin, abolishing the interactions with $\mathrm{W} 1$ by binding $\mathrm{Ca}^{2+}$ would allow a mobility to the $p$-hydroxybenzyl group, thus facilitating the breakdown reaction of the coelenterazine moiety and the rearrangement of the protein. Such a mechanism is supported by the fact that the luminescence of $h$-aequorin is 16 times more intense than that of native aequorin at the same concentration of $\mathrm{Ca}^{2+}$ (Shimomura et al. 1993b). In $h$-aequorin, the $p$-hydroxybenzyl group of coelenterazine moiety is substituted with a benzyl group, which does not form hydrogen bonds with a water molecule; in addition, the benzyl group makes no specific interactions with the residues in the binding pocket and it also causes less steric hindrance.

In the cases of $i-, b r-$, and $n$-aequorins containing coelenterazine analogues with bulky $\mathrm{C} 2$-substituents, their $\mathrm{Ca}^{2+}$-sensitivities and luminescence reaction rates are significantly lower than those of native aequorin, despite the fact that the stabilizing W1 is absent, and that no hydrophobic interactions occur between the 2-benzyl structure and the side chains of the binding pocket. Thus, the lower $\mathrm{Ca}^{2+}$-sensitivity and slower reaction rate of $i-, b r$-, and $n$ aequorins are probably due to the bulkiness of the 2-substituents. It is possible, however, that some interactions are formed between the aromatic group of the ligand and certain residues of the binding pocket in the course of the luminescence reaction. For example, the $\mathrm{C} 2$ benzyl group is close to His 169; if the aromatic ring were appropriately placed and rotated, it could form a stacking interaction with the imid- 
azole ring of His169. Prior to the light-emitting reaction, the position of the imidazole ring of His169 is fixed because its $\mathrm{N} \varepsilon 2$ atom forms a hydrogen bond with Tyr184. The C2 aromatic ring is also fixed at the same position in all structures of aequorins. Such conserved configurations are probably the best arrangement for protecting coelenterazine in the binding pocket until the occurrence of specific interactions in the process of the light-emitting reaction. Since Trp129 is also located close to the $\mathrm{C} 2$ aromatic ring, it might have some influence on the luminescence mechanism of aequorin. The halogen atoms of the $p$-iodobenzyl and $p$ bromobenzyl groups tend to break the $\pi-\pi$ interactions of the aromatic rings. It is possible that the lower $\mathrm{Ca}^{2+}$ sensitivities of $i$ - and $b r$-aequorins are caused not only by steric hindrance but also by the effect of the electronegative halogen atom on the benzyl group.

\section{C8 substitution}

In the molecule of native aequorin, the benzyl group at the C8 position of coelenterazine moiety is involved in a $\pi-\pi$ interaction with Trp108 of EF-hand III and a stacking interaction with Lys 39 of EF-hand I. In the case of $c p$ aequorin, however, the 8-cyclopentylmethy group of the ligand does not form these two hydrophobic interactions (Fig. 2A). Compared to native aequorin, $c p$-aequorin has a significantly higher $\mathrm{Ca}^{2+}$-sensitivity, and the half total time (time required to emit half its total light amount) is considerably shorter (Shimomura et al. 1993b), indicating an increase in the rate of the luminescence reaction. Similar increases in the $\mathrm{Ca}^{2+}$-sensitivity and reaction rate are also found with $c h$-aequorin, which contains a ligand containing 8-cyclohexylmethyl group (Shimomura et al. 1989).

The $\mathrm{Ca}^{2+}$ sensitivity and the reaction rate could be affected by the hydrophobic interactions that hinder the movement of the EF-hand helix, as well as by the steric hindrance due to the size of the 8 -substituent of the ligand. Compared to the benzyl group in native aequorin, the 8-cyclopentylmethyl group of $c p$-aequorin is smaller, whereas the 8-cyclohexylmethyl group in $c h$-aequorin is about the same or slightly larger. Despite the clear difference in the size of the 8-substituents, the $\mathrm{Ca}^{2+}$-sensitivities of $c p$-aequorin and $c h$-aequorin are nearly the same, and both are significantly higher than that of native aequorin (Shimomura et al. 1989, 1993b). Based on these facts, the absence of hydrophobic interactions, rather than the size of the 8-substituent, appears to be the main factor that makes these aequorins highly responsive to the structural change triggered by the binding of $\mathrm{Ca}^{2+}$.

$\mathrm{Ca}^{2+}$ sensitivity

The comparison of the loop structures of EF-hands I, III, and IV suggests that the first two EF-hands can bind $\mathrm{Ca}^{2+}$ more easily than EF-hand IV. In addition, all residues interacting with the 8- and 2-substituents of coelenterazine exist on EF-hands I and III, except Thr166. Although each EF-hand motif can be superimposed almost perfectly for all semi-synthetic aequorins and native aequorin, their $\mathrm{Ca}^{2+}$ sensitivities are widely different.

The manner by which the coelenterazine moiety is recognized by the residues in the binding pocket appears to be almost identical in all semi-synthetic aequorins and native aequorin. Since the binding pocket is made up of the helices of the EF-hands, the structural characteristics of each EFhand, such as the inter-helical angles and the relative positions, are also identical among all semi-synthetic aequorins and native aequorin. In coordinating the residues of the EF-hand loops to $\mathrm{Ca}^{2+}$, the $\mathrm{E}$ and $\mathrm{F}$ helices must move to the positions suitable for binding $\mathrm{Ca}^{2+}$. For this process to occur, the entire molecule must allow the movement of the EF-hands. In the molecule of aequorin, the helices of the four EF-hand motifs are attracted by hydrophobic forces to the coelenterazine moiety at the center of the molecule, together forming the active pocket. Under such a condition, almost all residues interacting with the coelenterazine moiety are those of the EF-hand helices. Therefore, the movement of the EF-hands and the associated changes of the protein, which take place upon the binding of $\mathrm{Ca}^{2+}$, must be under the influence of the pre-existing interactions between the EF-hand helices and coelenterazine moiety.

The luminescence reaction might be initiated as follows: $\mathrm{Ca}^{2+}$ binds to EF-hands I and III, thus changing the conformation of the coelenterazine-containing active site and breaking the hydrogen bond between the terminal oxygen of the peroxide of coelenterazine and Tyr184. It is possible that some interactions occur transiently between the protein and the coelenterazine moiety during the course of the rearrangement of protein structure that lead to the emission of light, although the protein structure eventually stabilizes in its $\mathrm{Ca}^{2+}$-bound form after the completion of the light-emitting reaction. From the present structures, it cannot be predicted what interactions would arise during the conformational change of the binding pocket.

Our structural study shows that altering the coelenterazine moiety of aequorin affects the various interactions involving the 2- and 8-substituents, which include the hydrophobic interaction, hydrogen-bonding interaction, and steric hindrance. In the aequorin molecule, the ability of EF-hands I and III to make a move in response to the binding of $\mathrm{Ca}^{2+}$ differs considerably depending on the structure of the coelenterazine involved; thus, semi-synthetic aequorins can show various different sensitivities to $\mathrm{Ca}^{2+}$.

\section{Conclusions}

The results of the present study indicate that the configurations of the coelenterazine moiety and the associated amino 
acid residues are highly conserved in semi-synthetic aequorins and native aequorin. There is no significant conformational difference between the EF-hand motifs of EF-hands I, III, and IV of the various aequorin molecules, despite the fact that semi-synthetic aequorins exhibit widely different luminescence characteristics. The structures presently described suggest that the modification of the $\mathrm{C} 2$ or $\mathrm{C} 8$ group of the coelenterazine moiety alters the interactions between these groups and their associated amino-acid residues in various different manners. In $c p$-aequorin, for example, the stacking and $\pi-\pi$ interactions of the $\mathrm{C} 8$ group are absent; therefore, $\mathrm{Ca}^{2+}$-induced conformational changes of protein occur more easily than in the case of native aequorin. In $b r$-, $i$-, and $n$-aequorin, the hydrogen bonding network mediated by $\mathrm{W} 1$ is absent but the steric hindrance of the $\mathrm{C} 2$-substituent impedes the relevant conformational changes. In the $\mathrm{Ca}^{2+}$-triggered luminescence reaction of aequorins, the first conformational change of protein takes place upon $\mathrm{Ca}^{2+}$ binding, followed by changes in the configuration of apoprotein, which eventually leads to the light emission. During this process, the configuration of the reaction pocket containing the coelenterazine moiety would also change, in accordance with various characteristics of the $\mathrm{C} 2$ - and $\mathrm{C} 8$ substitutent groups, including their size and aromaticity.

The C2-benzyl structure of the coelenterazine moiety is essential for the generation of active aequorin, although no specific interaction was found between the benzyl structure and amino acid residues in the aequorin structures. In $i$ - and $b r$-aequorin, the halogen atoms repel the $\pi-\pi$ interactions of the benzyl group and, being bulky, sterically hinder the conformational changes, features that perhaps underlie their lowered $\mathrm{Ca}^{2+}$ sensitivity.

There are two water molecules in the active site, $\mathrm{W} 1$ and $\mathrm{W} 2$. The present structures suggest that $\mathrm{W} 1$ stabilizes the $\mathrm{C} 2$-substituent, as found in the previous studies. The W2 molecule occupies an identical position in the structures of native aequorin and all semi-synthetic aequorins, and it might play some roles in the luminescence reaction. The amino-acid residues that recognize the imidazopyrazinone ring and the C6 substituent are also conserved, although we could not see any specific role for these residues beyond the stabilization of the coelenterazine moiety. It is possible, however, that these protein residues play certain important roles in the conformational change that follows the $\mathrm{Ca}^{2+}$ binding and also in the generation of aequorin, although the mechanisms involved would be difficult to predict from the present data.

The various types of intramolecular interactions observed in the present study are considered to be closely related to the $\mathrm{Ca}^{2+}$-sensitivities of aequorins, although they do not seem to be related to the capability of aequorin to emit light. After the first conformational change of aequorin triggered by the $\mathrm{Ca}^{2+}$-binding, the characteristics of the luminescence reaction are regulated by the specific interactions between the coelenterazine moiety and its associated protein residues. In order to develop a new aequorin with improved luminescence properties, attention should be focused on modifying these interactions, by the mutation of the protein as well as by changing the coelenterazine moiety.

\section{Materials and methods}

\section{Crystallization}

Semi-synthetic aequorins were prepared as previously reported (Shimomura et al. 1990). Crystals of the semi-synthetic aequorins were grown at $20^{\circ} \mathrm{C}$ by hanging drop vapor diffusion. Drops consisted of $2 \mu \mathrm{l}$ of protein solution $(10 \mathrm{mg} / \mathrm{ml})$ made in $10 \mathrm{mM}$ Bis-Tris ( $\mathrm{pH}$ 7.1) containing $2 \mathrm{mM}$ ethylenediaminetetraacetic acid and $2 \mathrm{M}$ ammonium sulfate. Crystals started to appear after $1 \mathrm{wk}$ and grew to a size suitable for diffraction experiments in the next several days.

\section{Data collection}

Data were collected on a PX210 CCD detector (Oxford Instruments) at BL44XU at SPring-8. The total rotation angle range for the $c p-, b r$-, and $i$-aequorin data was $180^{\circ}$, with a $1^{\circ}$ rotation interval in each exposure frame and with exposure times of $5 \mathrm{sec}$, $2 \mathrm{sec}$, and $2 \mathrm{sec}$ per frame, respectively. Three sets of different diffraction data were collected from $n$-aequorin crystals with different exposure times. The $n$-aequorin (data1) was collected at 1 sec per frame, with a total rotation angle range of $180^{\circ}$. $n$-Aequorin (data2) and $n$-aequorin (data3) were acquired in the following manner: Three data sets were collected from the same crystal with different position and exposure times $(2 \mathrm{sec}, 1 \mathrm{sec}$, and $7 \mathrm{sec}$ per frame) in sequence, covering total rotational ranges of $180^{\circ}, 345^{\circ}$, and $185^{\circ}$, respectively. $n$-Aequorin (data2) was prepared by merging using the $1 \mathrm{sec}$ per frame data as the lower resolution set $(43.5-3.0 \AA)$ and the $2 \mathrm{sec}$ per frame data as the higher resolution set $(3.5-1.6 \AA)$. $n$-Aequorin (data3) was prepared by merging the $1 \mathrm{sec}$ per frame (low resolution) set with the $7 \mathrm{sec}$ per frame (high

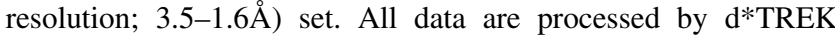
(Pflugrath 1999). Data collection and refinement statistics for all semi-synthetic aequorins are shown in Table 1.

\section{Structure determination and refinement}

Structure determination and refinement were performed using the CCP4 suite (Collaborative Computational Project Number4 1994). The structures were solved by molecular replacement with Molrep (Vagin and Teplyakov 1997) using native aequorin without coelenterazine as a search model. Refinements of the structures were performed using the program Refmac5 (Murshudov et al. 1997). At the first stage of the refinement, molecular orientations were refined by rigid body refinement. After several cycles of parameter refinements using non-crystallographic symmetry restraints, followed by model rebuilding using the program $\mathrm{O}$ (Jones et al. 1991), the position and conformation of the coelenterazine analogs were determined from the $\sigma_{\mathrm{A}}$-weighted $2 m \mathrm{Fo}-D \mathrm{Fc}$ map (Read 1986). The position of the terminal oxygen in the peroxide group of coelenterazine was determined using the $\sigma_{\mathrm{A}}$-weighted $m \mathrm{Fo}-D \mathrm{Fc}$ map (Read 1986), calculating the coelenterazine as a mono-oxide state. The geometry libraries of coelenterazine analogs were made 
Table 1. Crystallographic statistics for semisynthetic aequorin

\begin{tabular}{|c|c|c|c|}
\hline & $c p$-aequorin & $i$-aequorin & $b r$-aequorin \\
\hline Space group & $P 1$ & $P 1$ & $P 1$ \\
\hline \multicolumn{4}{|l|}{ Cell dimensions } \\
\hline$a, b, c(\AA)$ & $34.2,57.3,58.2$ & $34.2,54.3,57.4$ & $34.2,57.0,57.8$ \\
\hline$\alpha, \beta, \gamma\left({ }^{\circ}\right)$ & $72.2,80.0,68.41$ & $102.1,99.6,103.8$ & $72.2,80.3,68.0$ \\
\hline Wavelength $(\AA)$ & 0.9 & 0.9 & 0.9 \\
\hline Resolution $(\AA)$ & $33.5-1.8$ & $33.2-1.8$ & $33.3-1.8$ \\
\hline (outer shell) & $(1.86-1.80)$ & $(1.86-1.80)$ & $(1.86-1.80)$ \\
\hline No. of reflections & 36,134 & 35,601 & 35,673 \\
\hline Completeness $(\%)$ & $95.2(82.0)$ & $96.7(96.7)$ & $95.4(79.9)$ \\
\hline$l / \sigma(l)$ & $9.3(4.5)$ & $8.3(2.8)$ & $13.8(4.1)$ \\
\hline$R_{s y m}(\%)^{\mathrm{a}}$ & $5.1(13.8)$ & $7.7(14.2)$ & $5.4(13.1)$ \\
\hline$R^{\mathrm{b}} / R_{\text {free }}{ }^{\mathrm{c}}$ & $14.2 / 19.0$ & $18.8 / 25.0$ & $16.2 / 19.8$ \\
\hline \multicolumn{4}{|l|}{ R.m.s. deviation } \\
\hline Bond length $(\AA)$ & 0.019 & 0.021 & 0.017 \\
\hline Bond angle $\left({ }^{\circ}\right)$ & 1.81 & 1.9 & 1.678 \\
\hline \multicolumn{4}{|l|}{ Atoms per $\mathrm{AU}^{\mathrm{d}}$} \\
\hline Protein & 3020 & 3020 & 3020 \\
\hline Coelenterazine $^{\mathrm{e}}$ & 64 & 68 & 68 \\
\hline Water & 409 & 488 & 420 \\
\hline \multicolumn{4}{|l|}{ Ramachandran plot ${ }^{\mathrm{f}}$} \\
\hline Most favored $(\%)$ & 96.4 & 94.9 & 95.5 \\
\hline \multirow[t]{2}{*}{ Allowed $(\%)$} & 3.6 & 5.1 & 4.5 \\
\hline & $n$-aequorin (data1) & $n$-aequorin (data2) & $n$-aequorin (data3) \\
\hline Space group & $P 1$ & $P 1$ & $P 1$ \\
\hline \multicolumn{4}{|l|}{ Cell dimensions } \\
\hline$a, b, c(\AA)$ & $34.2,57.6,58.1$ & $34.5,57.5,58.1$ & $34.5,57.5,58.1$ \\
\hline$\alpha, \beta, \gamma(\AA)$ & $72.3,80.0,68.0$ & $72.3,80.0,68.0$ & $72.3,80.0,68.0$ \\
\hline Wavelength $(\AA)$ & 0.9 & 0.9 & 0.9 \\
\hline Resolution $(\AA)$ & $33.3-1.6$ & $33.3-1.6$ & $33.3-1.6$ \\
\hline (outer shell) & $(1.66-1.60)$ & $(1.66-1.60)$ & $(1.66-1.60)$ \\
\hline No. of reflections & 50,773 & 51,641 & 51,932 \\
\hline Completeness (\%) & $93.4(67.6)$ & $94.3(90.6)$ & $95.9(91.3)$ \\
\hline$l / \sigma(l)$ & $14.5(3.3)$ & $12.9(4.1)$ & $8.2(4.7)$ \\
\hline$R_{s y m}(\%)^{\mathrm{a}}$ & $4.4(14.3)$ & $8.8(25)$ & $7.8(8.0)$ \\
\hline$R^{\mathrm{b} / R_{\text {free }}{ }^{\mathrm{c}}}$ & $16.4 / 19.9$ & $17.9 / 21.5$ & $16.8 / 19.9$ \\
\hline \multicolumn{4}{|l|}{ R.m.s. deviation } \\
\hline Bond length $(\AA)$ & 0.014 & 0.023 & 0.016 \\
\hline Bond angle $\left({ }^{\circ}\right)$ & 1.542 & 2.081 & 1.663 \\
\hline \multicolumn{4}{|l|}{ Atoms per $\mathrm{AU}^{\mathrm{d}}$} \\
\hline Protein & 3046 & 2990 & 2990 \\
\hline Coelenterazine $e^{\mathrm{e}}$ & 74 & 74 & 72 \\
\hline Water & 496 & 279 & 276 \\
\hline \multicolumn{4}{|l|}{ Ramachandran plot ${ }^{\mathrm{f}}$} \\
\hline Most favored $(\%)$ & 95.9 & 96.1 & 96.4 \\
\hline Allowed (\%) & 4.1 & 3.9 & 3.4 \\
\hline
\end{tabular}

${ }^{a} R_{\text {sym }}=\sum|l-<l>| / \sum l$, where $l$ is the observed intensity, and $<l>$ is the averaged intensity of multiple symmetry-related observations of that reflection.

${ }^{\mathrm{b}} R=\sum\left\|F_{\text {obs }}|-| F_{\text {calc }}\right\| / \sum\left|F_{\text {obs }}\right|$, where $F_{\text {obs }}$ and $F_{\text {calc }}$ are the observed and calculated structure factors, respectively.

${ }^{\mathrm{c}} R_{\text {free }}=\sum\left\|F_{\text {obs }}|-| F_{\text {calc }}\right\| / \sum\left|F_{\text {obs }}\right|$ for $5 \%$ of the data not used at any stage of structural refinement.

${ }^{\mathrm{d}} \mathrm{AU}$ means asymmetric unit.

e "Coelenterazine" refers to coelenterazine analogs contained in each semi-synthetic aequorins. $c p$-Coelenterazine and $n$-coelenterazine (data3) were refined in the monoxide state.

${ }^{\mathrm{f}}$ Defined by PROCHECK (Laskowski 1993).

using a monomer library sketcher in the CCP4 suite (Collaborative Computational Project Number 4 1994). The iodine in $i$-coelenterazine and the bromine in $b r$-coelenterazine were refined with anisotropic temperature factors. The Ramachandran plot was calculated with PROCHECK (Laskowski et al. 1993), and the result is shown in Table 1. Superposition was performed with the program LSQKAB (Kabsch 1976). The crystallographic unit for all aequorins contained two molecules: molecule A and molecule B. Molecule A for all structures was superimposed onto the molecule A of $n$-aequorin (data1). 
Coordinates and structure factors have been deposited in the Protein Data Bank (http://www.pdb.org/), accession codes $c p$-aequorin: $1 \mathrm{UHH}, b r$-aequorin: $1 \mathrm{UHJ}, i$-aequorin: $1 \mathrm{UHI}$, and $n$-aequorin(data1): 1UHK.

\section{Acknowledgments}

We thank Prof. Tsukihara for his encouragement throughout this project, and all members of the X-ray crystallography group of the Institute for Protein Research for their support. A.N. is a member of the Structural Biology Sakabe Project (SBSP). This project is supported by a Grant-in-Aid for Centers of Excellence (COE) Research, a Grant-in-Aid for Scientific Research (no. 1169202), and the National Project on Protein Structural and Functional Analyses of the Ministry of Education, Culture, Sports, Science, and Technology of Japan.

\section{References}

Chattopadhyaya, R., Meador, W.E., Means, A.R., and Quiocho, F.A. 1992. Calmodulin structure refined at $1.7 \AA$ resolution. J. Mol. Biol. 228: $1177-$ 1192.

Collaborative Computational Project Number 4. 1994. The CCP4 Suite: Programs for protein crystallography. Acta Crystallogr. D Biol. Crystallogr. 50: $760-763$

Deng, L., Vysotski, E.S., Liu, Z.J., Markova, S.V., Malikova, N.P., Lee, J., Rose, J., and Wang, B.C. 2001. Structural basis for the emission of violet bioluminescence from a W92F obelin mutant. FEBS Lett. 506: 281-285.

Harris, M. and Jones, T.A. 2001. Molray-A web interface between $\mathrm{O}$ and the POV-Ray ray tracer. Acta Crystallogr. D Biol. Crystallogr. 57: 1201-1203.

Head, J.F., Inouye, S., Teranishi, K., and Shimomura, O. 2000. The crystal structure of the photoprotein aequorin at $2.3 \AA$ resolution. Nature 405: 372-376.

Jones, T.A., Zou, J.-Y., Cowan, S.W., and Kjeldgaard, M. 1991. Improved methods for the building of protein models in electron density maps and the location of errors in these models. Acta Crystallogr. A 47: 110-119.

Kabsch, W. 1976. A solution for the best rotation to relate two sets of vectors Acta Crystallogr. A 32: 922-923.

Kraulis, P.J. 1991. MOLSCRIPT: A program to produce both detailed and schematic plots of protein structures. J. Appl. Cryst. 24: 946-950.

Kuboniwa, H., Tjandra, N., Grzesiek, S., Ren, H., Klee, C.B., and Bax, A. 1995. Solution structure of calcium-free calmodulin. Nat. Struct. Biol. 2: 768-776.

Laskowski, R.A., MacArthur, M.W., Moss, D.S., and Thornton, J.M. 1993. PROCHECK: A program to check the stereochemical quality of protein structures. J. App. Cryst. 26: 283-291.
Liu, Z.J., Vysotski, E.S., Chen, C.J., Rose, J.P., Lee, J., and Wang, B.C. 2000 Structure of the $\mathrm{Ca}^{2+}$-regulated photoprotein obelin at $1.7 \AA$ resolution determined directly from its sulfur substructure. Protein Sci. 9: 2085-2093.

Liu, Z.J., Vysotski, E.S., Deng, L., Lee, J., Rose, J., and Wang, B.C. 2003. Atomic resolution structure of obelin: Soaking with calcium enhances electron density of the second oxygen atom substituted at the C2-position of coelenterazine. Biochem. Biophys. Res. Commun. 311: 433-439.

Merritt, E.A. and Bacon, D.J. 1997. Raster3D photorealistic molecular graphics. Method. Enzymol. 277: 505-524.

Murshudov, G.N., Vagin, A.A., and Dodson, E.J. 1997. Refinement of macromolecular structures by the maximum-likelihood method. Acta Crystallogr. D Biol. Crystallogr. 53: 240-255.

Pflugrath, J. 1999. The finer things in X-ray diffraction data collection. Acto Crystallogr. D Biol. Crystallogr. 55: 1718-1725.

Read, R.J. 1986. Improved Fourier coefficients for maps using phases from partial structures with errors. Acta Crystallogr. A 42: 140-149.

Shimomura, O. 1995. Luminescence of aequorin is triggered by the binding of two calcium ions. Biochem. Biophys. Res. Commun. 211: 359-363.

Shimomura, O. and Inouye, S. 1996. Titration of recombinant aequorin with calcium chloride. Biochem. Biophys. Res. Commun. 221: 77-81.

Shimomura, O. and Johnson, F.H. 1973. Chemical nature of light emitter in bioluminescence of aequorin. Tetrahedron Lett. 31: 2963-2966. 238

Shimomura, O., Johnson, F.H., and Saiga, Y. 1962. Extraction, purification and properties of aequorin, a bioluminescent protein from the luminous hydromedusan, Aequorea. J. Cell. Comp. Physiol. 59: 223-240.

Shimomura, O., Inoue, S., Johnson, F.H., and Haneda, Y. 1980. Widespread occurrence of coelenterazine in marine bioluminescence. Comp. Biochem. Physiol. 65B: 435-437.

Shimomura, O., Musicki, B., and Kishi, Y. 1988. Semi-synthetic aequorin. An improved tool for the measurement of calcium ion concentration. Biochem. J. 251: 405-410.

- 1989. Semi-synthetic aequorins with improved sensitivity to $\mathrm{Ca}^{2+}$ ions Biochem. J. 261: 913-920.

Shimomura, O., Inouye, S., Musicki, B., and Kishi, Y. 1990. Recombinant aequorin and recombinant semi-synthetic aequorins. Cellular $\mathrm{Ca}^{2+}$ ion indicators. Biochem. J. 270: 309-312.

Shimomura, O., Kishi, Y., and Inouye, S. 1993a. The relative rate of aequorin regeneration from apoaequorin and coelenterazine analogues. Biochem. J. 296 (Pt. 3): 549-551.

Shimomura, O., Musicki, B., Kishi, Y., and Inouye, S. 1993b. Light-emitting properties of recombinant semi-synthetic aequorins and recombinant fluorescein-conjugated aequorin for measuring cellular calcium. Cell Calcium 14: $373-378$.

Thomson, C., Herring, P., and Campbell, A. 1997. The widespread occurrence and tissue distribution of imidazolopyrazine luciferins. J. Biolumin. Chemilum. 12: 87-91.

Vagin, A. and Teplyakov, A. 1997. MOLREP: An automated program for molecular replacement. J. Appl. Cryst. 30: 1022-1025. 